\title{
Endoscopic Ultrasound-Guided Transgastric Fine Needle Aspiration of Splenic Metastasis
}

\author{
Avinash Bhat Balekuduru ${ }^{1}$ Aravind Subramani Kapali \\ Satyaprakash Bonthala Subbaraj' \\ ${ }^{1}$ Department of Gastroenterology, M.S. Ramaiah Memorial \\ Hospitals, Bangalore, Karnataka, India \\ ${ }^{2}$ Department of Surgical Oncology, M.S. Ramaiah Memorial \\ Hospitals, Bangalore, Karnataka, India \\ ${ }^{3}$ Department of Pathology, M.S. Ramaiah Memorial Hospitals, \\ Bangalore, Karnataka, India
}

J Digest Endosc:2020;11:238-241
Suneetha Parandhamaiah Kurella ${ }^{3}$

\author{
Abstract \\ Keywords \\ - endoscopic ultrasound \\ - splenic metastasis \\ - fine needle aspiration
}

\begin{abstract}
Address for correspondence Avinash Bhat Balekuduru, DM, Department of Gastroenterology, M.S. Ramaiah Memorial Hospitals, Bangalore 560054, Karnataka, India (e-mail: avinashbalekuduru@gmail.com).
\end{abstract}

\section{Introduction}

The word spleen comes from ancient Greek ođińv (splén) and is not commonly affected by disease. The differential diagnosis of splenic masses include malignancy (lymphoma, metastatic disease), infection (tuberculosis, fungal), and infiltrative processes such as sarcoidosis. ${ }^{1}$ Cross-sectional imaging can identify splenic masses, but they cannot differentiate the lesions. Histology is crucial but reserved only if there is no other safer accessible site to obtain tissue. ${ }^{2}$ Splenic tissue samples can be obtained either by surgery (splenectomy), percutaneous biopsy, or endoscopic ultrasound (EUS) guidance. ${ }^{3}$ The bleeding complication following percutaneous biopsy may be due to capsular laceration as a result of breathing. ${ }^{4}$ Utilizing proximity to the gastrointestinal tract, EUS can sample a splenic mass, traversing less tissue, and may reduce the risk of hemorrhage. ${ }^{5}$

Metastasis in the spleen (breast, lung, ovarian, colorectal cancers and melanomas) occurs in approximately $1 \%$ of malignant tumors. ${ }^{6}$ We present a rare case of a solitary splenic metastasis of transitional cell carcinoma of ovary, which occurred after a 4-year disease-free interval diagnosed by EUS-guided fine needle aspiration (FNA) biopsy.

\section{Case Report}

A 60-year-old woman presented with left upper quadrant pain for a duration of 5 days. In 2014, she was evaluated for right lower quadrant abdominal pain and fever for a duration of 30 days. She underwent staging laparotomy, total abdominal hysterectomy, and bilateral salpingo-oopherectomy for carcinoma ovary. She had received adjuvant chemotherapy with six cycles of paclitaxel and carboplatin. Follow-up imaging demonstrated complete remission of the disease. She had no other comorbidities or addictions. Her vital signs, physical examination, and laboratory results were unremarkable. Her blood cancer antigen 125 (CA125) level was $55.6 \mathrm{U} / \mathrm{mL}$ (reference range, $0-35 \mathrm{U} / \mathrm{mL}$ ).

Abdominal contrast-enhanced CT was done, which revealed an ill-defined minimally enhancing hypodense lesion measuring $47 \times 38 \mathrm{~mm}$ in size on the medial aspect of spleen (-Fig. 1). After obtaining written informed consent, EUS was performed using a Pentax linear echoendoscope- EG-3870 UTK connected to a Hitachi Avius estiva ultrasound machine (2012). On EUS examination, there was a $3.5 \times 3.3 \mathrm{~cm}$ heteroechoic mass lesion in the splenic hilum ( - Fig. 2). Transgastric EUS-guided FNA of the splenic lesion was done using a 22-G needle (EchoTip, Wilson-Cook, 


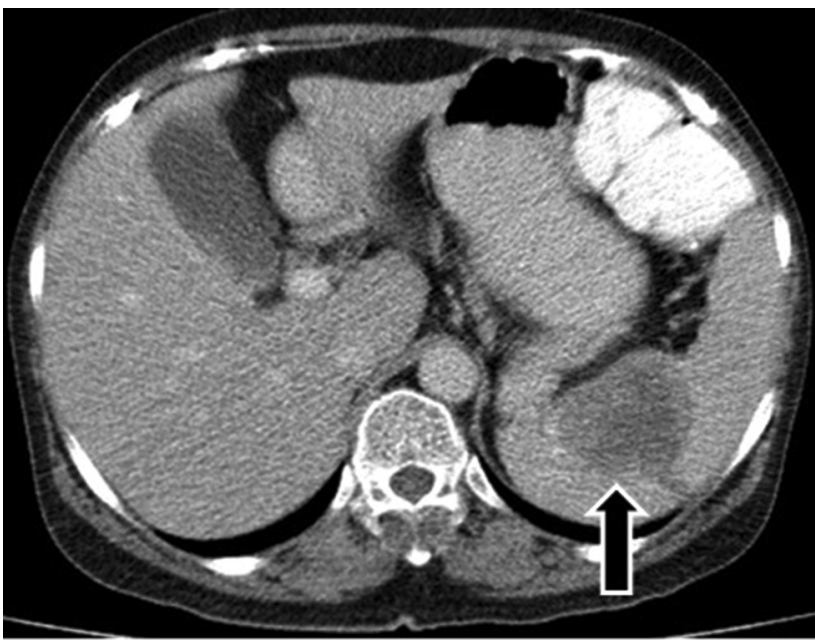

Fig. 1 Contrast-enhanced CT image showing ill-defined, minimally enhancing lesion in the spleen measuring $47 \times 38 \mathrm{~mm}$.

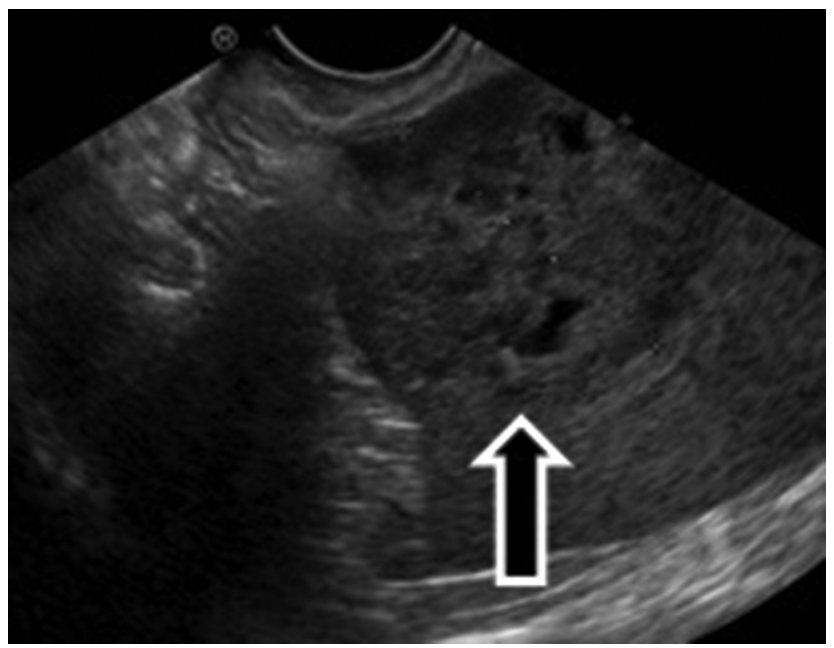

Fig. 2 EUS image showing $3.5 \times 3.3 \mathrm{~cm}$ heteroechoic mass lesion in the splenic hilum. EUS, endoscopic ultrasound.

Winston-Salem, North Carolina, USA). The biopsy needle is placed under ultrasound guidance down to the splenic capsule. The patient then was instructed to hold breath in order to bring the lesion into the field of view and the biopsy trajectory. The needle is advanced into the lesion and four passes with thread biopsy were taken. ${ }^{7}$ The needle was withdrawn and breathing resumed, decreasing the splenic capsular laceration/hematoma formation. Repeat EUS image showed contained needle tract hematoma ( - Fig. $\mathbf{3})$.

Histology revealed large atypical cells with hyperchromatic nuclei and moderate degree of pleomorphism ( - Fig. 4). Neoplastic cells showed positive immunohistochemical (ICH) staining for CK7 (-Fig. 5) and CA 125 (-Fig. 6) and were negative for CK 20 ( Fig. 7) and Uroplakin III ( Fig. 8). This immunophenotype is indicative of a metastatic primary transitional cell carcinoma of ovarian origin. She underwent uneventful open splenectomy, followed by adjuvant postop-

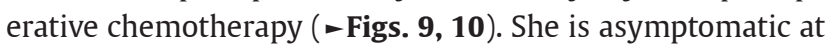
1 month follow-up.

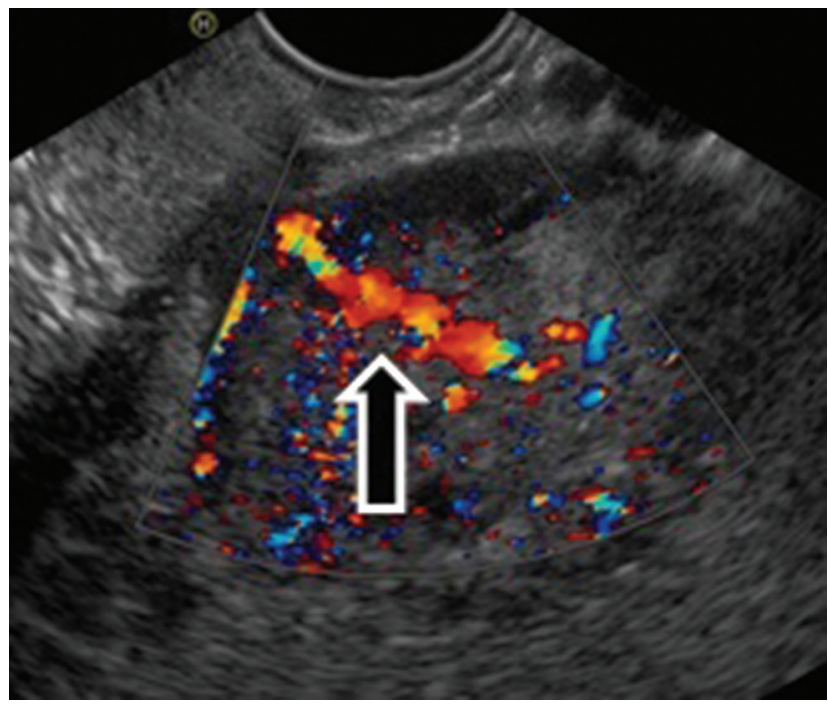

Fig. 3 EUS image showing contained needle tract hematoma. EUS, endoscopic ultrasound.

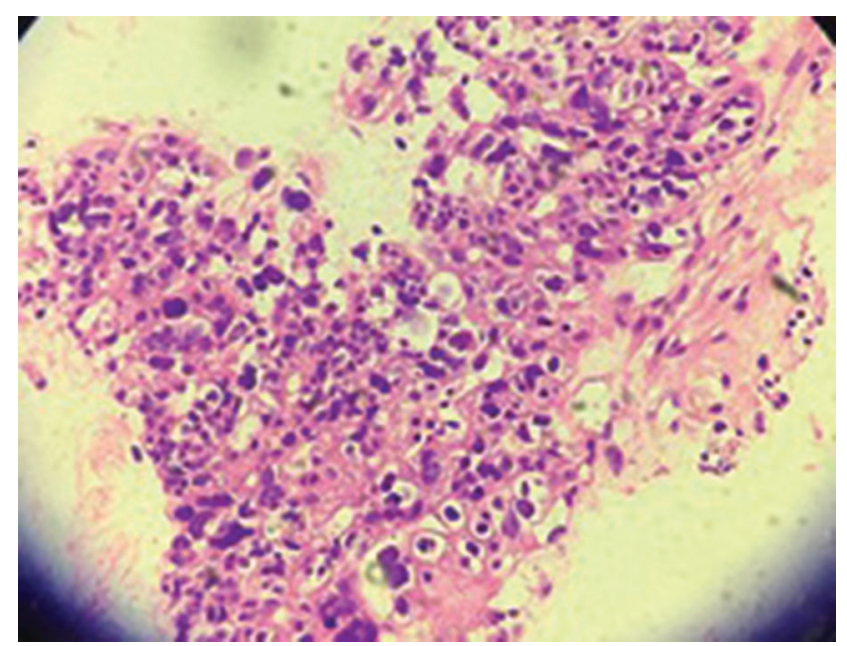

Fig. 4 Histology showed lymphocytes with large atypical cells, hyperchromatic nuclei, and moderate degree of pleomorphism.

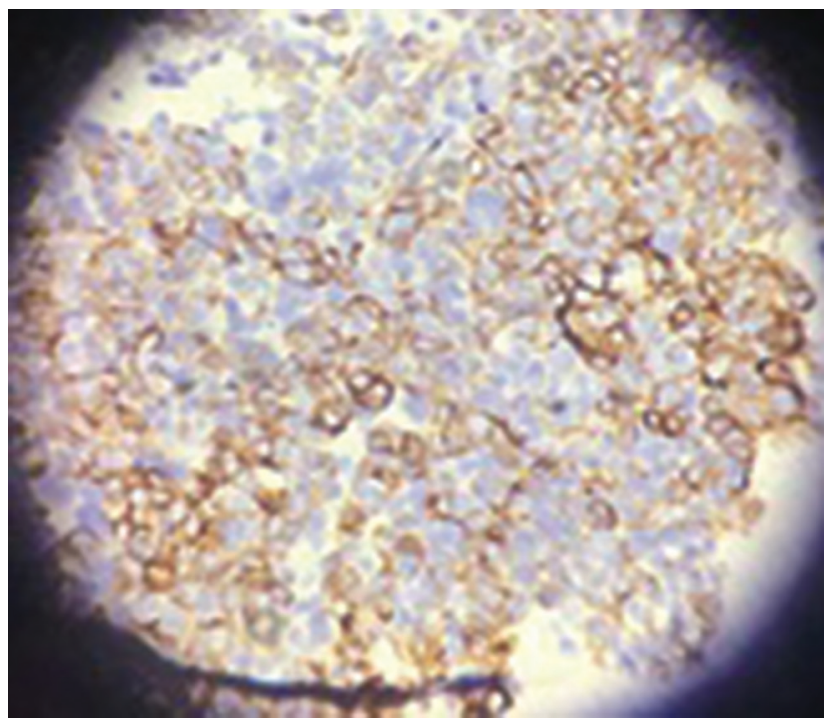

Fig.5 IHCtumorcells are positive for CK7.IHC, immunohistochemistry. 


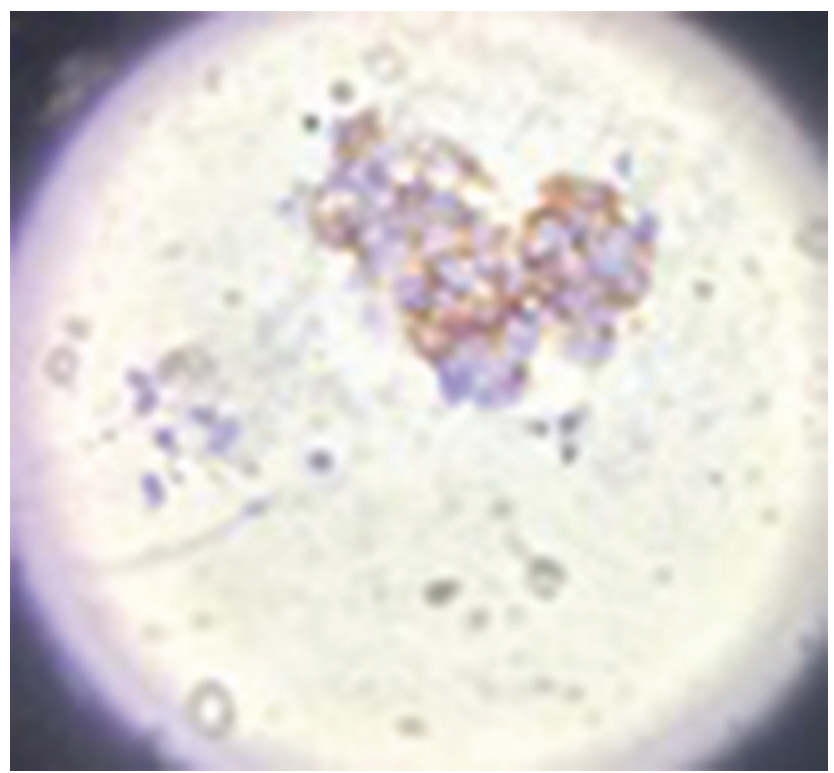

Fig. 6 IHC tumor cells are positive for CA125. IHC, immunohistochemistry,

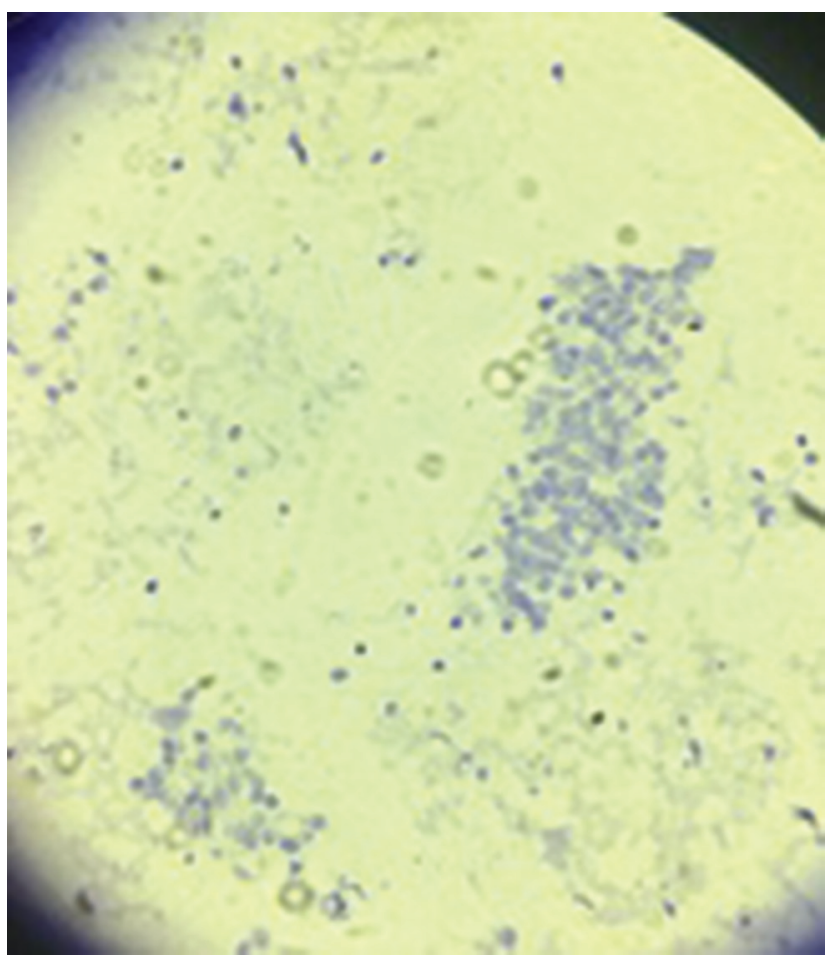

Fig. 7 IHC tumor cells are negative for CK20. IHC, immunohistochemistry.

\section{Discussion}

Most splenic metastases are accompanied by multivisceral tumor dissemination. The rarity of splenic metastases might be explained by the following reasons: (1) the poorly developed lymphoid system of the spleen, especially the lack of afferent lymphatic vessels, prevents the spleen from

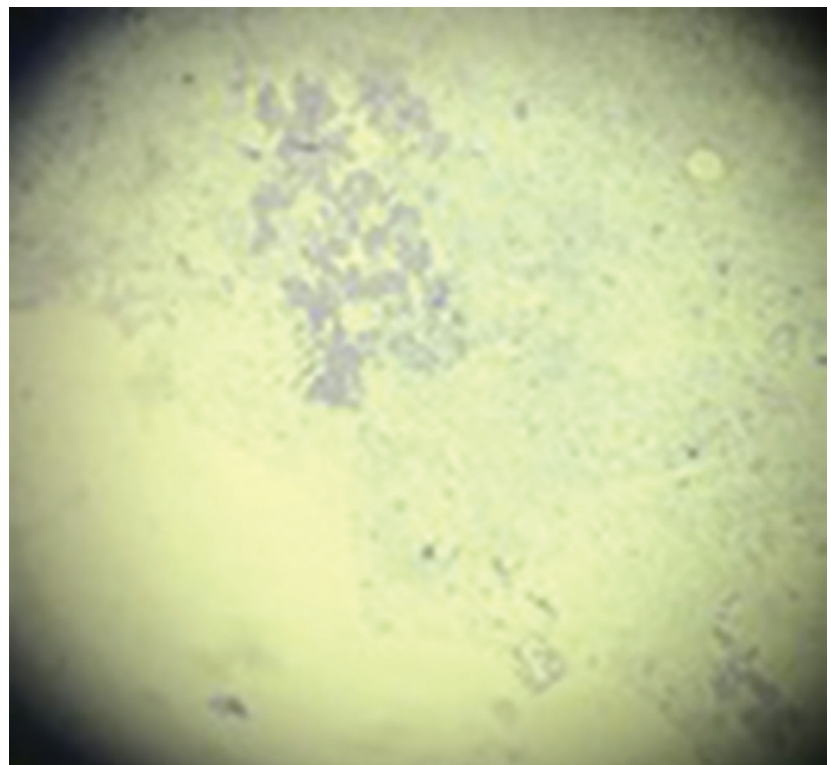

Fig. $8 \mathrm{IHC}$ tumor cells are negative for Uroplakin III. IHC, immunohistochemistry.

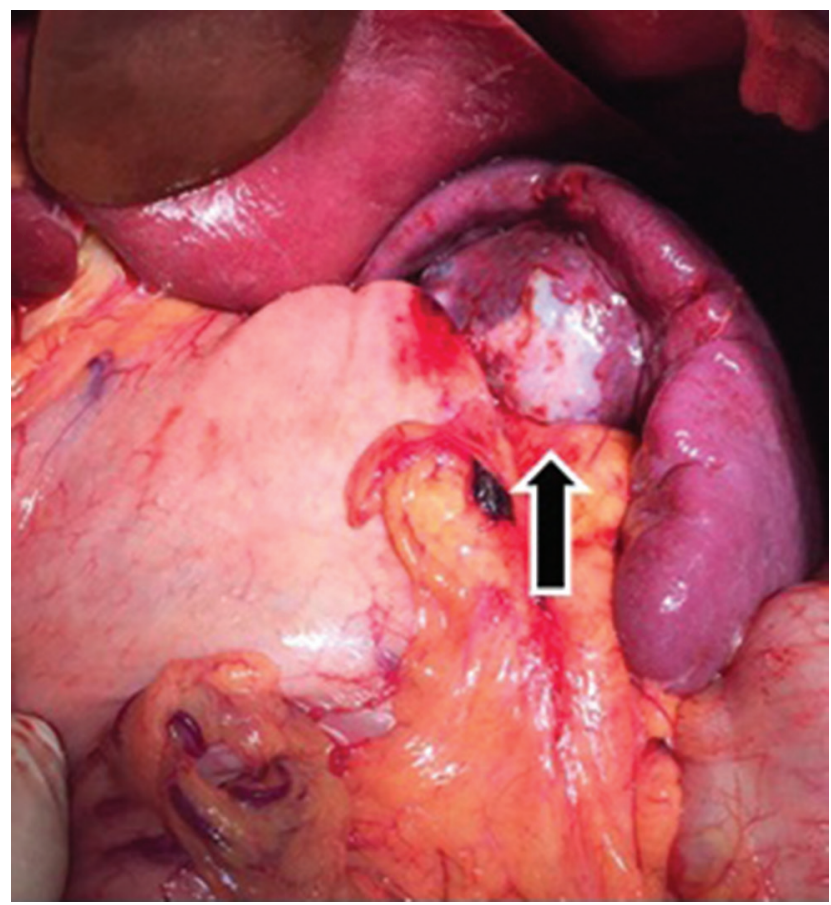

Fig. 9 Intraoperative picture showing splenic metastasis at hilum.

receiving metastatic tumor cells via the lymphatic route; (2) the sharp angle of splenic artery branching from the celiac trunk inhibits large clumps of tumor cells from passing through; and (3) the microenvironment of the spleen may hinder the growth of micrometastatic foci (characteristically present after a period of clinical latency of 11-120 months). ${ }^{6,7}$ F-FDG positron emission tomography can distinguish benign 


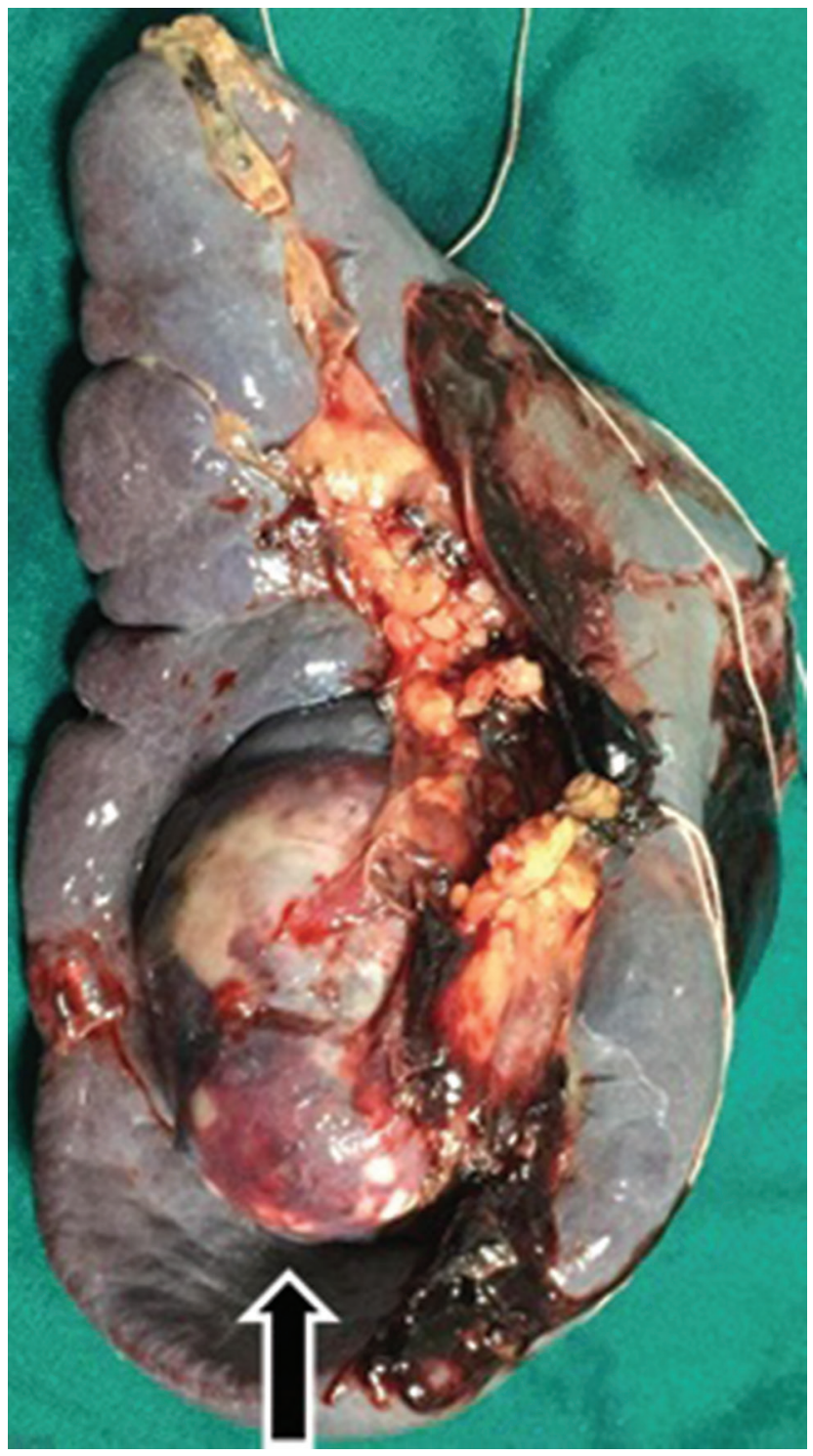

Fig. 10 Postoperative specimen of splenectomy showing splenic metastasis at hilum.

from malignant masses of the spleen, but it is preferable to obtain a tissue diagnosis. As compared with surgery with the highest morbidity at $8.2 \%$, image-guided percutaneous spleen biopsy has lower complication rate (4.2\%). EUS-guided splenic biopsy is an emerging modality for tissue acquisition. ${ }^{8}$

Peripheral lesion away from hilum will be targeted in image-guided biopsy with some intervening normal splenic tissue to minimize bleeding risk ${ }^{9}$. EUS is better in inaccessible splenic lesions that are located closely to hilum. ${ }^{10}$

Isolated splenic metastasis like in our patient points to a favorable prognosis. The tumor showed positivity for CK7 and CA 125 which are typical of female genital tract. As the primary was transitional cell carcinoma of ovary, CK20 and Uroplakin III were done, which were negative, ruling out bladder metastasis.
In conclusion, EUS provides good images of the spleen through the gastric wall and can acquire tissue from inaccessible splenic lesions that are located closely to hilum. EUS-guided FNA has been reported to be useful modality for cytopathological diagnosis of splenic lesions.

\section{Author Contributions}

Avinash B. was involved in the concept of case report, manuscript preparation, and final approval.

Aravind S. Kapali was involved in the manuscript preparation and final approval.

Suneetha K.P. was involved in the manuscript preparation and final approval.

SatyaPrakash B.S. was involved in the manuscript preparation and final approval.

\section{Declaration}

No grants were received for this study.

\section{Conflicts of Interest}

The authors declare that there are no conflicts of interest regarding the publication of this paper.

\section{References}

1 McInnes MD, Kielar AZ, Macdonald DB. Percutaneous image-guided biopsy of the spleen: systematic review and meta-analysis of the complication rate and diagnostic accuracy. Radiology 2011;260(3):699-708

2 Bastidas AB, Holloman D, Lankarani A, Nieto JM. Endoscopic ultrasound-guided Needle-Based Probe Confocal Laser Endomicroscopy (nCLE) of intrapancreatic ectopic spleen. ACG Case Rep J 2016;3(3):196-198

3 Singh AK, Shankar S, Gervais DA, Hahn PF, Mueller PR. Imageguided percutaneous splenic interventions. Radiographics 2012;32(2):523-534

4 Patel N, Dawe G, Tung K. Ultrasound-guided percutaneous splenic biopsy using an $18-\mathrm{G}$ core biopsy needle: our experience with 52 cases. Br J Radiol 2015;88(1055):20150400

5 Fritscher-Ravens A, Mylonaki M, Pantes A, Topalidis T, Thonke F, Swain P. Endoscopic ultrasound-guided biopsy for the diagnosis of focal lesions of the spleen. Am J Gastroenterol 2003;98(5):1022-1027

6 Resta G, Vedana L, Marino S, Scagliarini L, Bandi M, Anania G. Isolated splenic metastasis of ovaric cancer. Case report and literature review. G Chir 2014;35(7-8):181-184

7 Balekuduru AB, Dutta AK, Nagaruru SK, Sheik S, Kurella SP, Subbaraj SB. Comparison of diagnostic yield of endoscopic ultrasound-guided fine-needle aspiration cytology and cell block in solid lesions. J Dig Endosc 2017;8:176-181

8 Saab S, Challita Y, Holloman D, Hathaway K, Kahaleh M, Nieto $\mathrm{J}$. Case series review of the safety and efficacy of endoscopic ultrasound-guided splenic mass core biopsy. Clin Endosc 2018;51(6):600-601

9 Sammon J, Twomey M, Crush L, Maher MM, O'Connor OJ. Image-guided percutaneous splenic biopsy and drainage. Semin Intervent Radiol 2012;29(4):301-310

10 Rana SS, Bhasin DK, Rao C, et al. Splenic tuberculosis diagnosed by endoscopic ultrasound-guided fine needle aspiration. Endosc Ultrasound 2012;1(3):167-168 